mouse model of Myc-induced B-cell lymphomagenesis showed that kinetics of Mycinduced lymphoma in mice defective in specific components of the apoptotic pathway, such as caspase- 9 , are indistinguishable from those observed in the absence of p53 (ref. 12). The conclusions drawn from these studies have been bolstered by the existence of human tumor-derived p53 mutants compromised in apoptosis but not arrest function-arguing for the crucial importance of inactivating apoptotic functions during the course of human tumorigenesis.

Both cell cycle regulation and the induction of apoptosis are fundamental to the action of p53 in the mouse. Which activity is more relevant is probably context-dependent, with cell cycle regulation seeming to be key in thymocytes and apoptosis prevailing in other cell types, such as B cells. One prediction from Liu et al. is that mice deficient in p53 apoptotic target genes would not be prone to thymic lymphoma. The concept that apoptotic deficiency is not sufficient for thymic lymphomagenesis represents a change from previous thinking; because thymocytes undergo a clear p53-dependent apoptotic response on DNA damage ${ }^{13}$, there is a longstanding belief that the predisposition of $\mathrm{p} 53$ deficient mice to thymic lymphoma results from defects in this apoptotic program.

These studies also raise the question of why $C d k n 1 a^{-/-}$mice, which have a compromised G1 arrest response, are not prone to thymic lymphoma. This condition may relate to the fact that p21-deficient cells are only partially defective in the G1 arrest checkpoint, and that the activity of another, unknown p53-regulated cell cycle arrest target gene can provide sufficient cell cycle regulation to prevent the malignant transformation of thymocytes. Another, not mutually exclusive, explanation for limited tumorigenesis in $C d k n 1 a^{-/-}$mice is developmental or somatic compensation by functionally related cyclindependent kinase inhibitors, similar to what has been described recently for the Rb family ${ }^{14}$.

\section{Vive la différence}

Among the remaining issues is the question of what triggers p53 activation to prevent thymic lymphoma development. p53 is activated by any of a number of stresses, including DNA damage, hyperproliferative signals and hypoxia. The signals that trigger p53 activation and, by extension, the downstream pathway activated by p53 may be fundamentally different in mice and humans. For example, unlike mouse cells, which have very long telomeres, human cells have relatively short telomeres and therefore suffer severe consequences on telomere attrition. Telomere-based crisis, in which critically short telomeres result in breakage-fusionbridge cycles, is accompanied by p53 activation and a prominent apoptotic response in many human cell types ${ }^{15}$. In certain human cell types, there may be a strong selection pressure specifically against the apoptotic function of $\mathrm{p} 53$, which could explain how human tumor mutants in which the apoptotic function has been inactivated might have arisen. A prediction of this idea would be that the $\operatorname{Trp} 53^{515 C}$ allele in the context of telomere-based crisis would render mice very cancer-prone in certain compartments, a hypothesis that is now testable through the use of telomerase-deficient $\operatorname{Tr} p 53^{515 \mathrm{C} / 515 \mathrm{C}}$ compound mutant mice.

p53 itself is a difficult target for cancer therapy given its active role in promoting aging when inappropriately activated. Consequently, depending on the tumor type, it may be most productive to selectively target only a specific aspect of p53 function to mollify accelerated aging in normal tissues, particularly in the setting of conventional therapy. Defining the specific signaling surrogates involved in tumor maintenance is a good first step toward designing well-tolerated and effective therapies for the many cancers related to p53 mutations.

1. Hanahan, D. \& Weinberg, R.A. Cell 100, 57-70 (2000).

2. Vousden, K.H. \& Lu, X. Nat. Rev. Cancer 2, 594-604 (2002).

3. Liu, G. et al. Nat. Genet. 36, 63-68 (2004).

4. Ludwig, R.L., Bates, S. \& Vousden, K.H. Mol. Cell. Biol. 16, 4952-4960 (1996).

5. Attardi, L.D. \& Jacks, T. Cell. Mol. Life Sci. 55, 48-63 (1999).

6. Brugarolas, J. et al. Nature 377, 552-557 (1995).

7. Deng, C., Zhang, P., Harper, J.W., Elledge, S.J. \& Leder, P. Cel/ 82, 675-684 (1995).

8. Martin-Caballero, J., Flores, J.M., Garcia-Palencia, P. \& Serrano, M. Cancer Res. 61, 6234-6238 (2001).

9. Morgenbesser, S.D., Williams, B.O., Jacks, T. \& DePinho, R.A. Nature 371, 72-74 (1994).

10. Symonds, H. et al. Cell 78, 703-711 (1994).

11. Yin, C., Knudson, C.M., Korsmeyer, S.J. \& Van Dyke, T. Nature 385, 637-640 (1997).

12. Schmitt, C.A. et al. Cancer Cell 1, 289-298 (2002).

13. Lowe, S.W., Schmitt, E.M., Smith, S.W., Osborne, B.A. \& Jacks, T. Nature 362, 847-849 (1993).

14. Sage, J., Miller, A.L., Perez-Mancera, P.A., Wysocki, J.M. \& Jacks, T. Nature 424, 223-228 (2003).

15. Chin, L. et al. Cel/ 97, 527-538 (1999).

\title{
White meat or dark?
}

\section{Ava E Brent \& Clifford J Tabin}

\section{In zebrafish, slow twitch muscle is specified from a somitic muscle precursor pool by local Hedgehog (Hh) signals. A new study identifies the transcription factor Blimp-1 as a key downstream mediator in this process.}

\begin{abstract}
"White meat or dark?" is a familiar question asked around holiday tables. But what exactly makes meat white or dark? Dark meat, composed primarily of slow twitch muscle fibers, is specialized for extended exertions (standing, walking, slow swimming) and gets the

Ava E. Brent and Clifford J. Tabin are in the Department of Genetics, Harvard Medical School, 77 Avenue Louis Pasteur, Boston, Massachusetts 02115, USA.

e-mail: tabin@genetics.med.harvard.edu
\end{abstract}

consistent energy it needs from its high myoglobin content. By contrast, fast muscle, the main component of white meat, fuels quick bursts (sprinting, short flights) and contains less myoglobin.

Because chickens and turkeys stand or roam about and rarely fly, their leg meat is dark while their breast and wing meat is white. Conversely, wild birds, such as ducks, which frequently fly, have dark breast and wing meat. As your holiday guests feast on their turkey, this may be enough information for them. But for authors Philip Ingham, Sudipto Roy and colleagues, the question of white meat or dark led to a fascinating investigation into how slow and fast muscle is established during zebrafish embryogenesis and to the identification of a genetic switch responsible for determining muscle fiber fate ${ }^{1}$.

\section{Instructive cues}

In the vertebrate embryo, skeletal muscle arises from somites, segmented blocks of mesoderm lying on either side of the notochord and 
neural tube. Signals from surrounding tissues induce the somitic mesoderm to generate distinct compartments, the sclerotome and myotome, from which the axial skeleton and associated axial muscle emerge. Fish, which rely primarily on large muscle blocks for swimming, have somites consisting largely of myotome, with a relatively small ventral sclerotome population (Fig. 1d). Fate maps show that medial (adaxial) cells abutting the notochord and expressing the muscle determining factors myoD and myf5 generate slow muscle, whereas lateral cells generate fast muscle (Fig. 1a). Adaxial cells begin expressing genes specific to slow fibers and then migrate radially away from the notochord to form a superficial slow muscle layer surrounding the initially lateral population of fast muscle precursors ${ }^{2}$ (Fig. 1c,d).

Notochord signals direct adaxial cells to commit to both a general myogenic and a specific slow twitch fiber fate ${ }^{3}$, and loss- or gainof-function experiments identified these signals as members of the Hh family. In particular, mutations in $\mathrm{Hh}$ pathway components, such as Sonic Hedgehog (Shh) and Smoothened (Smo; a transmembrane protein that transduces the Hh signal), impede slow muscle formation ${ }^{4,5}$, whereas overexpression of Shh switches myotomal cells from fast to slow fiber fate ${ }^{6-8}$. But although a role for $\mathrm{Hh}$ signaling in slow muscle induction is clear, the identity of genes acting downstream of Hh in muscle precursors is not.

\section{Bring in the Blimp}

Now, through positional cloning of a zebrafish mutant called u-boot (ubo), the authors have identified Blimp-1, a transcription factor previously implicated in B-cell development ${ }^{9,10}$, as a good candidate. Like Hh pathway mutants, ubo embryos show loss of slow muscle development, with cells initially fated for slow fiber switching to fast. Notably, although the presomitic mesoderm in $u b o$ mutants is competent to respond to $\mathrm{Hh}$ signaling (as evidenced by expression of the Hh target genes myoD and $p t c 1$ ), there is no specification of slow muscle even after overexpression of Shh ${ }^{11}$.

In the present work, the authors show that mutations of Blimp-1 underlie the slow muscle defects seen in $u b o$ mutants. Blimp-1 is expressed specifically in adaxial cells before expression of slow muscle-specific genes but after the onset of expression of myoD and myf5, suggesting that the muscle precursors influenced by $u b o$ are already committed to a myogenic lineage (Fig. 1b). Expression of Blimp-1 then declines before the slow muscle precursors begin their radial migration, suggesting that $u b o$ may initiate, but not maintain, slow muscle fate.

Consistent with its proposed role as a slow muscle regulator downstream of Hh signaling, adaxial expression of $u b o$ is lost in smo mutants, and overexpression of Shh produces ectopic expression of $u b o$. Moreover, overexpression of $u b o$ itself is sufficient to cause fast muscle precursors to change fate and become slow muscle. To test whether it is loss of $u b o$ that leads to loss of slow fiber differentiation in the absence of Hh signaling, the authors showed that overexpression of $u b o$ in smo mutants is sufficient to rescue slow muscle development ${ }^{1}$. This experiment in particular shows that it is through $u b o$ that $\mathrm{Hh}$ signaling directs adaxial myotomal cells to adopt a slow fiber fate.

\section{Context is everything}

The Hh pathway patterns a wide variety of tissues, from invertebrate embryonic segments and imaginal discs to the vertebrate central nervous system and myotome. How does one signal control so many outcomes? The answer probably lies in the tissue-specific responses that the Hh signal elicits, namely, the particular genes activated or repressed in target cells that give the Hh signal a tissue-specific meaning. Now, with the identification of one such gene, $u b o$, we gain valuable insight into one particular Hh-induced response: the

Figure 1 Successive stages in commitment of adaxial myotome to slow muscle fate. Transverse sections through presomitic mesoderm (a-c) or somite (d) at progressively more anterior levels. Myotome is shown in red, sclerotome in blue, notochord in yellow and neural plate (NP) or neural tube (NT) in gray. (a) Adaxial myotome cells (ovals) surrounding the notochord begin expressing myoD and myf5 (pink), indicating commitment to a myogenic program. Sclerotome cells occupy a ventral position. (b) In response to $\mathrm{Hh}$ secreted from the notochord (arrows), $u b o$ (purple) is activated in the same adaxial cells that express myoD and myf5. (c) After activation of $u b o$, adaxial cells begin expressing slow muscle-specific genes, such as slow myosin heavy chain (MyHC; green), suggesting slow muscle differentiation. Expression of $u b o$ is then turned off as adaxial cells begin a radial migration (arrows in $\mathbf{c}$ ) to their final position as an outer, superficial layer of slow muscle, still expressing slow myosin heavy chain (d). Some adaxial cells (the muscle pioneer cells) remain close to the notochord (green ovals in $\mathbf{d}$ ). The sclerotome migrates dorsally to surround the notochord, and the remaining myotome differentiates into fast muscle (red). specification of slow muscle fibers. Notably, the gene mandating fiber-type specification in the myotome also functions as a 'selector' gene in an entirely unrelated contextthe differentiation of $\mathrm{B}$ lymphocytespresumably in response to a different signal. That the myotome responds to $\mathrm{Hh}$ induced $u b o$ by differentiating into slow muscle and not B lymphocytes is probably because these cells are already committed to becoming muscle precursors before $u b o$ is even turned on, thereby restricting other outcomes.

This study raises several questions. For instance, $u b o$ acts as a transcriptional repressor in the context of B-cell differentiation ${ }^{12}$. Does it do the same during myotome patterning (perhaps repressing expression of fast fiber-specific genes), and if so, what are its targets? It will also be important to determine if there is a similar pathway of slow fiber specification at work in the somites of higher terrestrial vertebrates. In fish, most of the somite forms myotome, and myotome ends up nearest the notochord; in higher vertebrates, where the axial skeleton has a more central role, it is sclerotome that surrounds the notochord and is patterned by it. Although ventral midline Shh certainly has a role in myotome development in higher vertebrates $^{13}$, it is unclear whether that role includes specification of fiber type ${ }^{14}$.

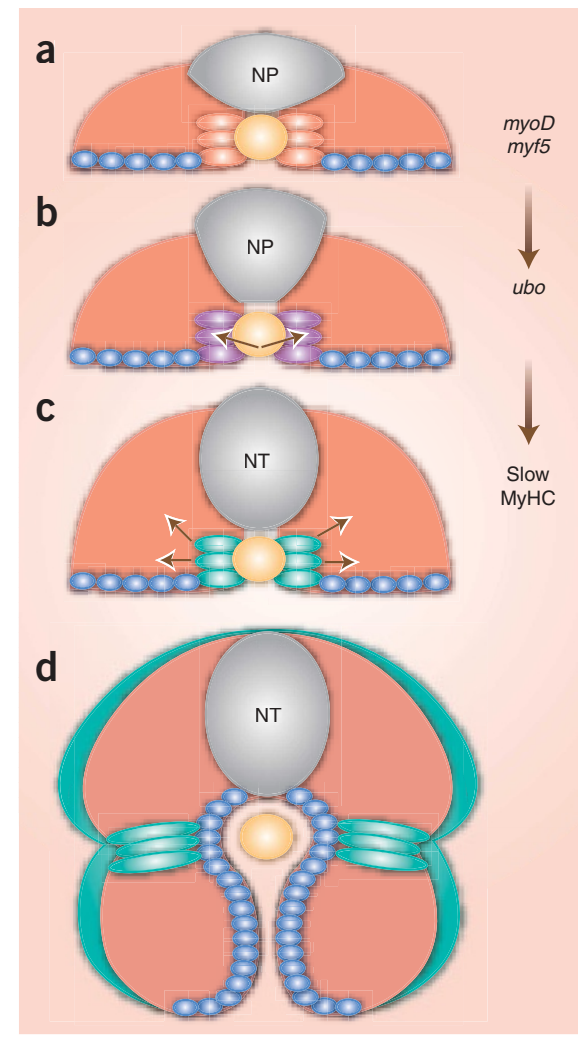


Nonetheless, there are reports that mouse homolog of Blimp-1 is expressed in the myotome ${ }^{15}$, and it will be interesting to determine whether it functions as a slow muscle selector there as well.

1. Baxendale, S. et al. Nat. Genet. 36, 88-93 (2004)

2. Devoto, S.H. et al. Development 122, 3371-3380 (1996).

3. Weinberg, E.S. et al. Development 122, 271-280
(1996)

4. Lewis, K.E. et al. Dev. Biol. 216, 469-480 (1999).

5. Barresi, M.J. Stickney, H.L. \& Devoto, S.H. Development 127, 2189-2199 (2000).

6. Currie, P.D. \& Ingham, P.W. Nature 382, 452-455 (1996).

7. Blagden, C.S. et al. Genes Dev. 11, 2163-2175 (1997).

8. Wolff, C., Roy, S. \& Ingham, P.W. Curr. Biol. 13, 1169-1181 (2003).

9. Turner, C.A. Jr., Mack, D.H. \& Davis, M.M. Cell 77, 297-306 (1994).
10. Shapiro-Shelef, M. et al. Immunity 19, 607-620 (2003).

11. Roy, S., Wolff, C. \& Ingham, P.W. Genes Dev. 15 1563-1576 (2001)

12. Schebesta, M., Heavey, B. \& Busslinger, M. Curr. Opin. Immunol. 14, 216-223 (2002).

13. Brent, A.E. \& Tabin, C.J. Curr. Opin. Genet. Dev. 12 548-557 (2002).

14. Sacks, L.D. et al. Development 130, 3391-3402 (2003).

15. Chang, D.H., Cattoretti, G. \& Calame, K.L. Mech. Dev. 117, 305-309 (2002)

\section{Two Pax are better than one}

\section{Richard S Mann}

In fly eye development, new experiments suggest that two distinct $P a x$ genes control tissue growth and identity, respectively. Notably, these two functions may be encoded by distinct isoforms of the human gene PAX6

Most biologists, and many nonbiologists, are probably familiar with the experiment in which scientists generated fruit flies that had more than the normal pair of compound eyes $^{1}$. This result, produced by the forced misexpression of the gene eyeless (ey; called PAX6 in humans), was so notable that it found its way to the front page of The New York Times with the headline "Science Outdoes Hollywood", an accolade that has set a new standard for genetics research ${ }^{2}$. Since this first set of discoveries, the mechanism underlying the control of eye development by the master control gene ey has been the subject of intense research. How can a single gene instruct the development of such a complex structure as the fly's compound eye? On page 31 of this issue, a report by Maria Dominguez and her colleagues ${ }^{3}$ provides new insight into this problem.

\section{Sizing up the eyes}

To generate a complex organ, such as a compound eye, the correct cell types must be formed and these cell types must be correctly organized. In other words, an organ-specific identity must be generated. ey (or Pax6), together with a cohort of subordinate transcription factors, is essential for this aspect of eye development ${ }^{4}$. Organ size must also be controlled, and size control has to be coordinated with the process of organ-identity specification. For the fly eye, there is some

Richard S. Mann is in the Department of Biochemistry and Molecular Biophysics, Center for Neurobiology and Behavior, Columbia University, 701 West $168^{\text {th }}$ Street, HHSC 1104, New York, New York 10032, USA.

e-mail: rsm10@columbia.edu controversy as to whether growth and identity are controlled by the same pathway or by different pathways. Most researchers agree that growth of the eye is controlled by the Notch signal transduction pathway, which is activated during eye development at the interface between the dorsal and ventral halves of the developing eye, also called the dorsal-ventral organizer $^{5-7}$. Some researchers have suggested that Notch also controls eye identity and $e y$ expression $^{8,9}$. Others, however, argue that Notch does not control ey expression or eye identity, only the growth of the eye ${ }^{10}$. That Notch is not responsible for eye identity makes more sense, because this pathway is used not only in the eye but repeatedly during development in a wide variety of contexts. But these results raise an important question: if not $e y$, what are the Notch targets that mediate its growth-promoting activity in the eye?

The new work by Dominguez et al. ${ }^{3}$ answers this question. Their experiments suggest that growth and identity specification are separable pathways in fly eye development. They identify the Pax6-like gene eyegone $(\text { eyg })^{11}$ as the Notch target responsible for mediating its growth-promoting effects in the eye (Fig. 1). Consistent with this notion, in the absence of eyg, the eye primordium does not grow, even though ey is still expressed. This is notable, in part because eyg is not expressed throughout the eye but seems to be

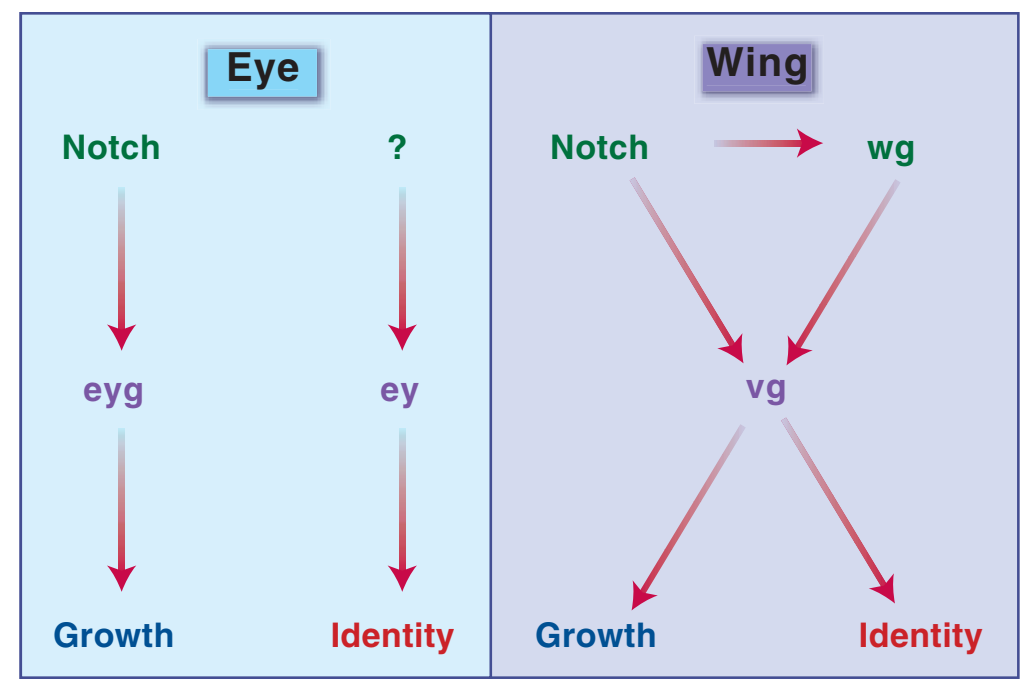

Figure 1 In the fly eye, tissue growth is regulated by Notch and its target eyg. Eye identity is regulated by ey. In the wing, the gene vg carries out both functions and is regulated by both the Notch and Wingless $(\mathrm{Wg})$ pathways. 